\title{
Design of a UV-responsive microactuator on a smart device for light-induced ON-OFF-ON motion
}

\author{
Meng Xiao, Chao Jiang and Feng Shi \\ Combining a stimuli-responsive material and the Marangoni effect, a microactuator containing a ultraviolet (UV)-light-responsive \\ photoresist and surfactant is fabricated. The locomotion of a functionally cooperating device loaded with the microactuator can \\ be initiated by irradiation with $365 \mathrm{~nm}$ UV light, ceased by the removal of the UV light and restarted by re-exposure to the UV \\ light. Moreover, the device exhibits good direction control via selective irradiation of photoresponsive microactuators at \\ designated locations. This work is the first example using the chemical Marangoni effect to produce photoresponsive ON-OFF- \\ ON motion of a macroscopic object on water surfaces with little impact on the experimental environment and opens up new \\ opportunities for the design of novel advanced functional materials with controlled properties.
}

NPG Asia Materials (2014) 6, e128; doi:10.1038/am.2014.76; published online 19 September 2014

\section{INTRODUCTION}

The movement of centimeter-scale objects on water surfaces has numerous potential applications, such as mimicking the movement of insects, exploring the complexity of creatures and researching fluid drag. ${ }^{1-5}$ However, challenges remain in locomoting centimeter-scale objects on water surfaces because their sizes are too small to load a power system. Normally, the locomotion of such macroscopic objects should address two issues: overcoming the drag force caused by the surface effect and achieving motion from a limited power supply. To overcome the drag force, drag-reducing coatings with superhydrophobic properties were introduced to reduce the fluidic friction and improve the efficiency of the power system. ${ }^{2,6}$ To address the power supply issue, two strategies have been developed. One is to apply an external field as the driving force, ${ }^{7,8}$ which is limited by the specific properties of the materials and is strongly dependent on manual control. The other solution is to introduce an external material flow to provide chemical power, such as the decomposition of hydrogen peroxide ${ }^{4,9-19}$ or the reduction of protons by zinc ${ }^{20,21}$ for bubble propulsion or other propelling mechanisms, the Marangoni effect caused by a surface tension gradient ${ }^{22-24}$ or biomotors such as myosins, kinesins and dyneins via the hydrolysis of adenosine triphosphate. ${ }^{25}$ As a branch of material flow research, the Marangoni effect is a facile, environmentally benign and rapidly responsive method for driving small objects automatically.

The Marangoni effect, a common phenomenon in nature caused by a surface tension gradient, is widely used to drive the locomotion of insects such as the beetles of the genus Stenus. ${ }^{26,27}$ The driving force is induced by mass transfer along an interface between two types of liquids owing to a surface tension gradient caused by different compositions or temperatures. ${ }^{28}$ Inspired by this phenomenon, scientists have applied the Marangoni effect as self-propelling power for the locomotion of macroscopic objects on interfaces. Yoshikawa and his co-workers ${ }^{29}$ investigated the spontaneous motion of a solidliquid composite induced by the chemical Marangoni effect when an oil droplet attached to solid soap is placed on water. Velev and his coworkers ${ }^{30}$ presented the gel-based self-propelled particles moving in an oscillatory mode propelled by the Marangoni effect. However, most of these movements are uncontrolled, that is, once placed on the water surface, the object moves in a random manner. ${ }^{31-34}$ To promote spontaneous motions from an uncontrolled to a guided manner, we fabricated a smart device that remained immobile under acidic conditions and started moving because of the Marangoni effect when the $\mathrm{pH}$ was adjusted to alkali conditions, thus mimicking the locomotion process of beetles of the genus Stenus. ${ }^{24}$ Although this method can successfully start motion in a controlled manner (ON motion), challenges still remain in the following two aspects: achieving a controllable ON-OFF-ON motion that can be switched on, off and then back on again; ${ }^{35}$ shortening the switching time because adjusting the $\mathrm{pH}$ of the entire system in the above work is time consuming. To address these issues, Okawa et al. ${ }^{36}$ developed a system that could convert light into thermal energy and further induced the locomotion of a polydimethylsiloxane object via a thermal Marangoni effect. Baigl and his co-workers ${ }^{37,38}$ demonstrated the light-responsive smart motion of a droplet by a wavelength-dependent liquid/liquid interfacial gradient, and this approach can be used for precise manipulation of a floating oil droplet. To the best of our knowledge, there is no report on fast

State Key Laboratory of Chemical Resource Engineering and Key Laboratory of Carbon Fiber and Functional Polymer, Ministry of Education, Beijing University of Chemical Technology, Beijing, People's Repubic of China

Correspondence: Professor F Shi, State Key Laboratory of Chemical Resource Engineering and Key Laboratory of Carbon Fiber and Functional Polymer, Ministry of Education, Beijing University of Chemical Technology, 15 Beisanhuan East Road, Chaoyang District, Beijing 100029, People's Republic of China.

E-mail: shi@mail.buct.edu.cn

Received 28 April 2014; revised 17 July 2014; accepted 23 July 2014 
intelligent ON-OFF-ON motion of a macroscopic object caused by a chemical Marangoni effect responding to light.

Herein, we designed a smart device capable of ON-OFF-ON locomotion in response to 365-nm ultraviolet (UV) light. This device has two components: a superhydrophobic boat with drag-reducing properties and a UV-responsive microactuator, which is a silicon wafer covered with a mixture of photoresist and surfactant. When placed on a developer solution, the device floated and remained motionless on the water surface. After the UV-responsive microactuator at the back of the superhydrophobic boat was exposed to 365-nm UV light, the photoresist began to decompose, and the wrapped surfactant in the mixture diffused onto the water surface, generating a chemical Marangoni effect and propelling the device forward. The movement could be halted by turning off the UV light and restarted by turning the light back on. By attaching several photoresponsive microactuators to different side surfaces of the boat, we could control the movement direction of the device by selectively irradiating these microactuators with UV light. The as-prepared smart device not only performed the ON-OFF-ON motion in rapid response to photostimuli with little impact on the experimental environment but also exhibited directional selectivity via the selective irradiation of different photoresponsive microactuators.

\section{EXPERIMENTAL PROCEDURE}

\section{Materials and methods}

The materials used in the study were $\mathrm{HAuCl}_{4}$ (Sinopharm Chemical Reagent, Beijing, China), nickel foam (Anping Xinlong Wire Mesh Manufacture, Anping, China), $\mathrm{HS}\left(\mathrm{CH}_{2}\right)_{11} \mathrm{CH}_{3}$ (Sigma-Aldrich, Shanghai, China) and positive photoresist (RZJ-304) (Suzhou Ruihong Electronic Chemical, Suzhou, China). Scanning electron microscopy (SEM) was performed with a ZEISS SUPRA 55 (Carl Zeiss AG, Jena, Germany) at $20.0 \mathrm{kV}$. Atomic force microscopy (Dimension 3100 from Veeco Instruments, Plainview, NY, USA) was used to characterize the morphology and thickness of the surface coating. All the movies were recorded with a SONY HDR-790E (Sony Corporation, Tokyo, Japan).

The surfactants used were BYK-088, BYK-141, BYK-371 and BYK-3700 (Bike Chemical (tongling), Tongling, China), whose chemical natures are solutions of foam-destroying polymers and polysiloxanes. The above BYK family surfactant has good solubility with the photoresist of RZJ-304 that we used. BYK-141 was selected as the optimal surfactant based on motion experiments in Section S1 of Supplementary Information.

\section{Fabrication of the superhydrophobic boat}

As illustrated in Scheme 1, first, nickel foam was folded into a trapezoid boat $\left(2.1 \times 1.2 \times 1.3 \mathrm{~cm}^{3}\right)$ and cleaned three times by alternating ultrasonication in ethanol and deionized water. Second, the boat was immersed in an aqueous solution of $\mathrm{HAuCl}_{4}\left(4 \mathrm{mg} \mathrm{ml}^{-1}\right)$ for $30 \mathrm{~min}$, washed with deionized water and dried in an oven. Finally, the dried boat was coated with $\mathrm{HS}\left(\mathrm{CH}_{2}\right)_{11} \mathrm{CH}_{3}$ by chemical vapor deposition at room temperature for $10 \mathrm{~h}$.

\section{Fabrication of the UV-responsive microactuator}

Silicon wafers $\left(1 \times 1.2 \mathrm{~cm}^{2}\right)$ were immersed in a piranha solution $\left(\mathrm{H}_{2} \mathrm{O}_{2}: \mathrm{H}_{2} \mathrm{SO}_{4}=1: 3\right.$ by volume) for $\sim 60 \mathrm{~min}$, washed with deionized water three times, dried with nitrogen gas and heated on a hot plate $\left(100^{\circ} \mathrm{C}\right)$ for $5 \mathrm{~min}$. Then, a spin coater was used to spin a mixture of photoresist and surfactant (89:11 in volume ratio) on the silicon wafers at 600 r.p.m. for $18 \mathrm{~s}$ and at 2000 r.p.m. for 20 s. Next, the silicon wafers covered with a mixture of photoresist and surfactant were heated on a hot plate at $100{ }^{\circ} \mathrm{C}$ for $90 \mathrm{~s}$ to volatilize the solvent in the photoresist mixture. The entire fabrication process was performed in the dark. After exposure to UV irradiation, the photoresist could be washed away by a developer of $\mathrm{NaOH}$ (aq., $1 \mathrm{M}$ ). The UV-responsive microactuator was attached to designated positions of the superhydrophobic boat using double-sided tape, forming the smart device capable of ON-OFFON motions.

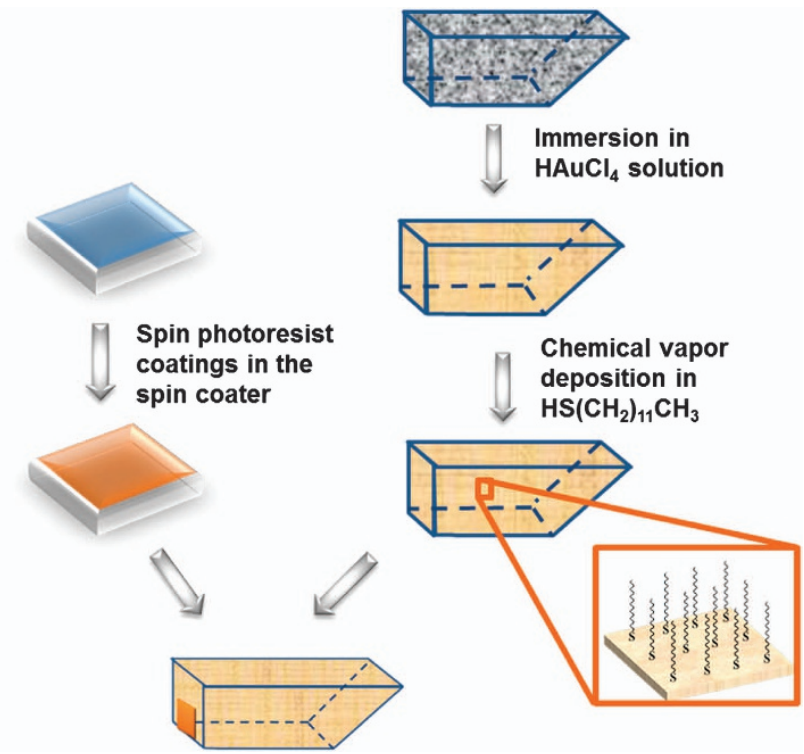

Scheme 1 Illustration of the fabrication of the smart device.

\section{RESULTS AND DISCUSSION}

SEM was used to characterize the surface morphology of the nickel foam before/after the electroless metal deposition and the photoresponsive microactuator at various irradiation times. As shown in Figure 1a, the untreated nickel foam was a porous film composed of continuous mesh structures with an average diameter of $\sim 400 \mu \mathrm{m}$. After electroless metal deposition in $\mathrm{HAuCl}_{4}$ solution, the surface was covered with three-dimensional gold aggregates with nanoscale branches (Figures $1 \mathrm{~b}$ and c). This deposition provided sufficient roughness, and superhydrophobicity was exhibited after the structure was modified with a self-assembled monolayer of $\mathrm{HS}\left(\mathrm{CH}_{2}\right)_{11} \mathrm{CH}_{3}$. The superhydrophobicity can be interpreted using the Cassie mode, ${ }^{39}$ that is, $\cos \theta_{\mathrm{r}}=f_{1} \cos \theta_{1}+f_{2} \cos \theta_{2}$, where $\theta_{\mathrm{r}}$ is the apparent contact angle, $f_{1}$ and $f_{2}$ are the proportions of liquid-solid and liquid-air contacting areas, respectively, and $f_{1}+f_{2}=1, \theta_{1}$ and $\theta_{2}$ are the intrinsic contact angles of the liquid-solid and air-solid, respectively. Taking $\theta_{1}$ as a constant for a specific surface and $\theta_{2}$ as $\pi$, we can obtain a positive correlation of $\cos \theta_{\mathrm{r}}=\cos \theta_{1}-\left(\cos \theta_{1}+1\right) f_{2}$, meaning that an increased liquid-air contacting area $\left(f_{2}\right)$ is favorable for improved superhydrophobicity (high $\theta_{\mathrm{r}}$ ). The hierarchical structure for increased roughness provides an efficient strategy to increase the liquid-air contacting area. ${ }^{40} \mathrm{In}$ addition, the modification of the lowsurface-energy coating of $\mathrm{HS}\left(\mathrm{CH}_{2}\right)_{11} \mathrm{CH}_{3}$ is favorable for reducing $\theta_{1}$. Hence, the rough gold aggregates and $\mathrm{HS}\left(\mathrm{CH}_{2}\right)_{11} \mathrm{CH}_{3}$ coating should contribute to superhydrophobicity.

Moreover, we used SEM to characterize the surface morphology of the UV-responsive microactuator before and after irradiation with 365-nm UV light. From Figure 1d, we can see that before UV irradiation, the mixture of the photoresist and surfactant formed a flat film on the silicon wafer. After irradiating the film at $7.4 \mathrm{~mW} \mathrm{~cm}^{-2}$ for $3 \mathrm{~s}$ and washing it with a developer, the entire surface had a rough morphology, indicating that the photoresist had decomposed homogeneously (Figure 1e). When the irradiation time was increased to $9 \mathrm{~s}$, the surface roughness increased, indicating that the etched depth of the mixture of the photoresist and surfactant increased with the duration of UV irradiation (Figure 1f). Figures $1 \mathrm{~g}-\mathrm{i}$ present the corresponding atomic force microscopy images for Figures $1 \mathrm{~d}-\mathrm{f}$, from which we can determine that before irradiation, the photoresist 

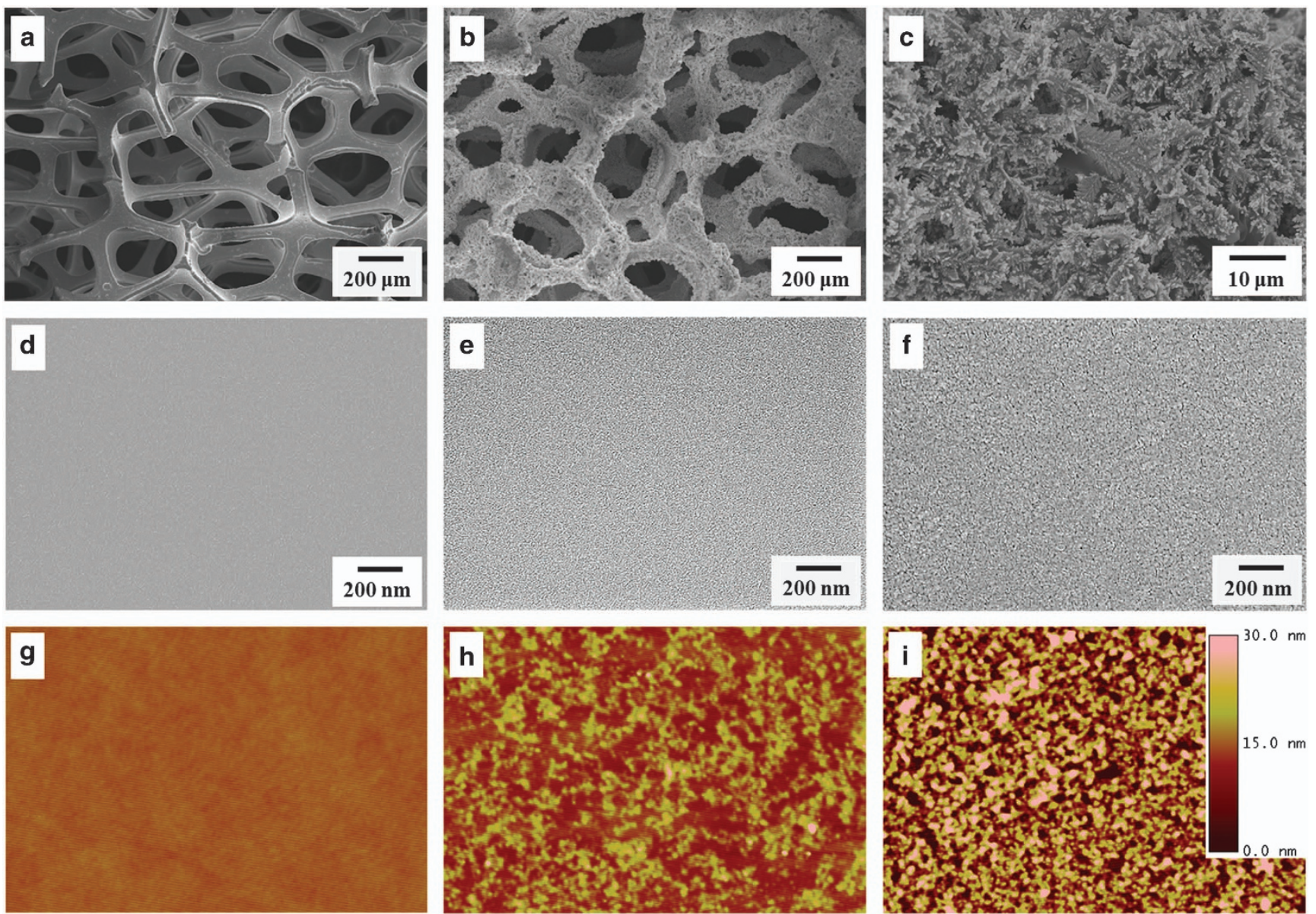

Figure 1 Scanning electron microscopy (SEM) images of nickel foam (a) before and (b) after deposition of gold structures; (c) magnified image of (b). SEM images of the photo-responsive microactuator (d) before and after irradiation under exposure doses of (e) $22.2 \mathrm{~mJ} \mathrm{~cm}^{-2}$ and (f) $66.6 \mathrm{~mJ} \mathrm{~cm}^{-2}$; (g-i) the corresponding atomic force microscopy (AFM) images $\left(5 \times 3.5 \mu \mathrm{m}^{2}\right)$ of $(\mathbf{d}-\mathbf{f})$.

mixture displayed a smooth surface with a surface roughness of $0.56 \pm 0.01 \mathrm{~nm}$. With increased irradiation time, the roughness increased to $2.87 \pm 0.10 \mathrm{~nm}$ at an exposure dose of $22.2 \mathrm{~mJ} \mathrm{~cm} \mathrm{~cm}^{-2}$ and then $7.96 \pm 0.33 \mathrm{~nm}$ at $66.6 \mathrm{~mJ} \mathrm{~cm}^{-2}$. These results strongly support the phenomenon that the etched depth of the mixture of the photoresist and surfactant increased with the duration of UV irradiation. The decomposition of the photoresist contributed to the release of surfactant, which generated a surface tension gradient on the water surface for the locomotion of the device.

After surface modification of the superhydrophobic boat with dragreducing properties and attachment of a photoresponsive microactuator at the stern of the boat, the smart device moved in response to $365-\mathrm{nm} \mathrm{UV} \mathrm{light} \mathrm{at} 72.6 \mathrm{~mW} \mathrm{~cm}^{-2}$ for $3 \mathrm{~s}$. Note that the absorbance of the photoresist mixture ranges from 200 to $440 \mathrm{~nm}$, that is, the smart device should move after the UV irradiation with a wavelength below $440 \mathrm{~nm}$ (for details, see Section S2 in Supplementary Information). As demonstrated in Figure 2a, the device moved in a straight line at an average speed of $3 \mathrm{~cm} \mathrm{~s}^{-1}$ after UV irradiation, whereas before the device was irradiated, the device floated essentially motionlessly on the water surface. When the device was turned in another direction using tweezers, the device continued to move in a straight line at a similar speed (for details, see Supplementary Movie S1). The motion behavior of the smart device is interpreted as follows: when the photoresponsive microactuator was irradiated by UV light, the photoresist decomposed, and the surfactant underneath was released, which had a surface tension of $\sim 65 \mathrm{mN} \mathrm{m}^{-1}$ (Supplementary Figure S3). Therefore, the difference in the surface tension between water $\left(72 \mathrm{mN} \mathrm{m}^{-1}\right)$ and the released surfactant could reach $7 \mathrm{mN} \mathrm{m}^{-1}$, which led to the surface tension gradient and further Marangoni effect. Considering that a liquid with lower surface tension tends to move toward liquid with a higher surface tension, in this case, the local solution with the surfactant at the end of the smart device would flow to water area behind the device. Therefore, according to Newton's law of equal and opposite reactions, the smart device must in turn move forward. After the device stopped in the middle of the trough, we exposed the device to UV irradiation again and observed almost no further motion. This phenomenon likely occurred because all of the photoresist in the mixture had decomposed and the surfactant had been released thoroughly, which led to the disappearance of the local surface tension gradient and thus prevented the restarting of the smart device.

To visualize the physical-chemical mechanism of the motion, we recorded the diffusion process of the surfactant on the water surface with thymol blue as an indicator. We spread thymol blue powder on the water surface, which is insoluble and formed a layer of the indicator. Then, a smart device was placed on the surface and irradiated by UV light, as shown in Figure 3. In the absence of UV light, the device remained static, and the water surface almost 
a

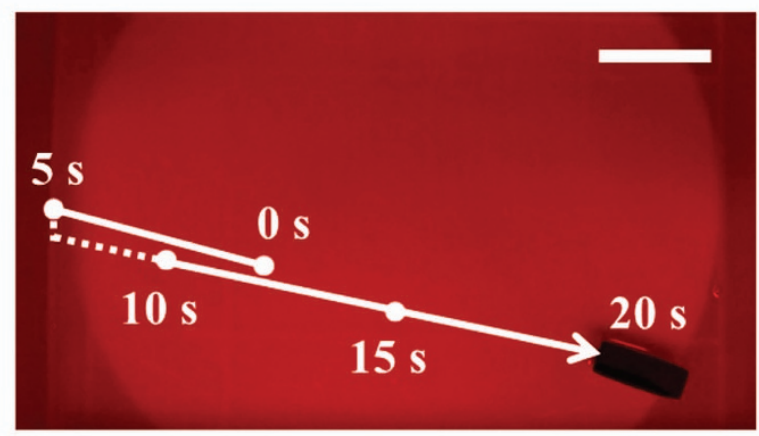

C

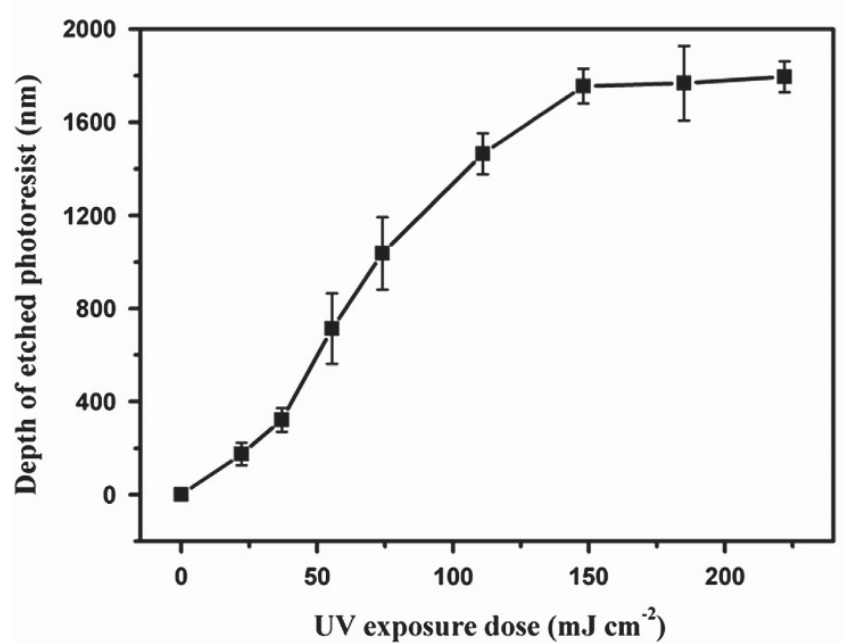

b

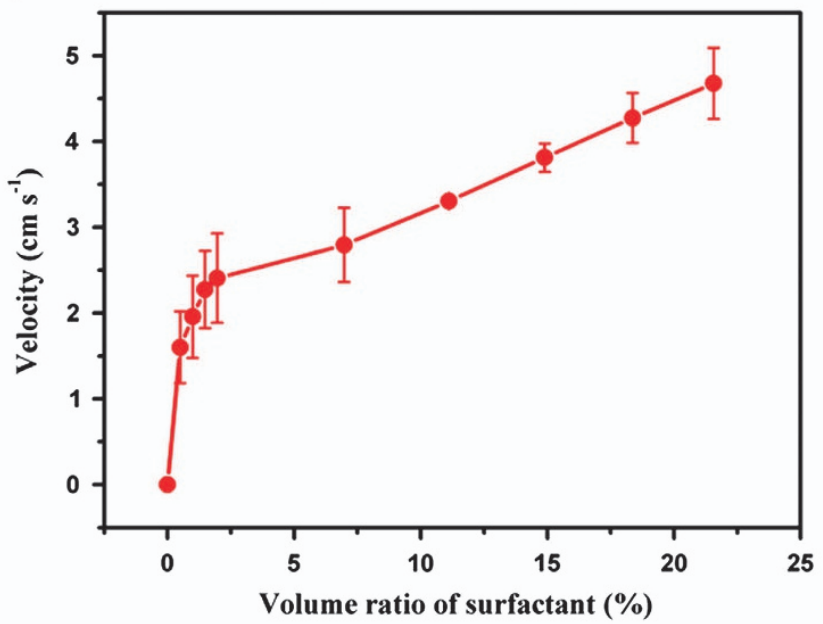

d

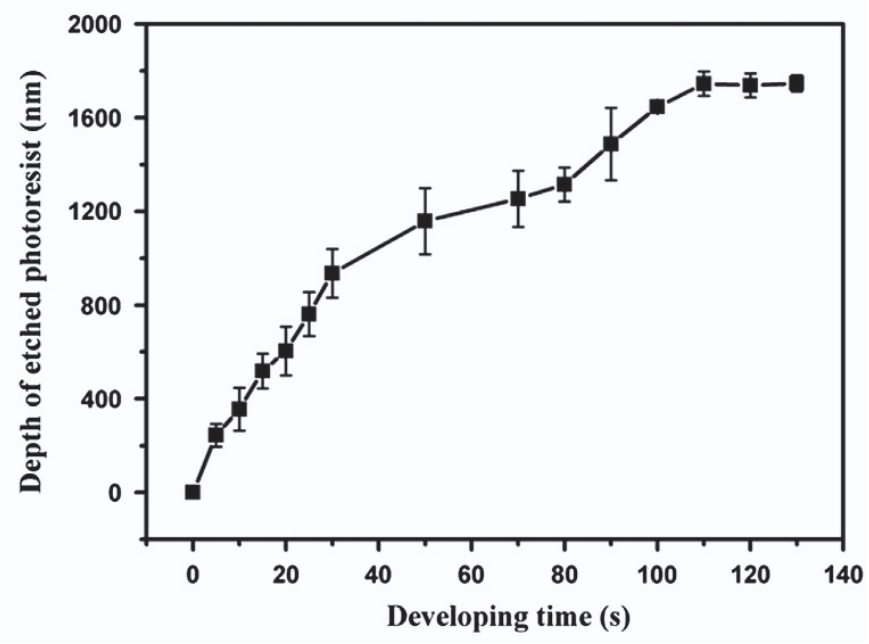

Figure 2 (a) The position of the smart device was recorded at 5-s intervals (white dots and solid lines; the dashed line represents the turning process with tweezers), as shown in Supplementary Movie S1. Scale bar $=5 \mathrm{~cm}$. (b) The moving velocity of the smart device versus the volume ratio of the surfactant (BYK-141). The depth of the etched photoresist versus (c) ultraviolet (UV) exposure dose and (d) developing time in the developer when the volume ratio of the surfactant was fixed at $11 \%$.

experienced no changes (Figure 3a). Without UV irradiation, the photoresist did not decompose and prevented release of the surfactant, and thus no surface tension gradient occurred for the locomotion of the device. After being exposed to UV irradiation in Figure 3b, a small circle of water appeared at the end of the device where the UV light was applied. The appearance of this circular water surface was attributed to the flow from the low-surface-energy area to the highsurface-energy area, which was caused by the surface tension gradient generated from the released surfactant. In addition, the smart device was propelled and moved forward. With the locomotion of the device, a large water circle grew behind the device by repelling thymol blue particles, which was caused by the continuous release of the surfactant, as illustrated in Figures $3 \mathrm{c}$ and $\mathrm{d}$. To exclude the effect of the dissolution process after the photoresist was decomposed on the motion of the device, we performed control experiments under similar experimental conditions but with no surfactant in the system; the results are presented in Supplementary Movie S2. The boat remained static upon exposure to UV irradiation under identical conditions, demonstrating that the dissolution of the photoresist in the basic solution had no effect on the velocity of the device; hence, the locomotion of the smart device in Supplementary Movie S1 was attributed mainly to the chemical Marangoni effect caused by the release of the surfactant.

To clarify the effect of the volume ratio of the surfactant in the mixture on the velocity of the boat, we investigated the correlation between the movement velocities and the volume ratio. As demonstrated in Figure 2b, the velocity increased sharply with an increasing volume ratio when the volume ratio of the surfactant was below 2.5\%. Above this value, however, the increase in velocity with increased volume ratio slowed because the surfactant gradually reached its critical micelle concentration at this point, resulting in a small surface tension gradient and thus preventing sharp increases in velocity. This result corresponded well with Curve III in Traube's rule ${ }^{41}$ namely, the surface tension decreases remarkably with increasing surfactant concentration at low concentrations and almost levels off at high concentrations (Section S3 in Supplementary Information). Note that the ON-OFF-ON controllability of the locomotion of the device decreased as the velocity increased. 

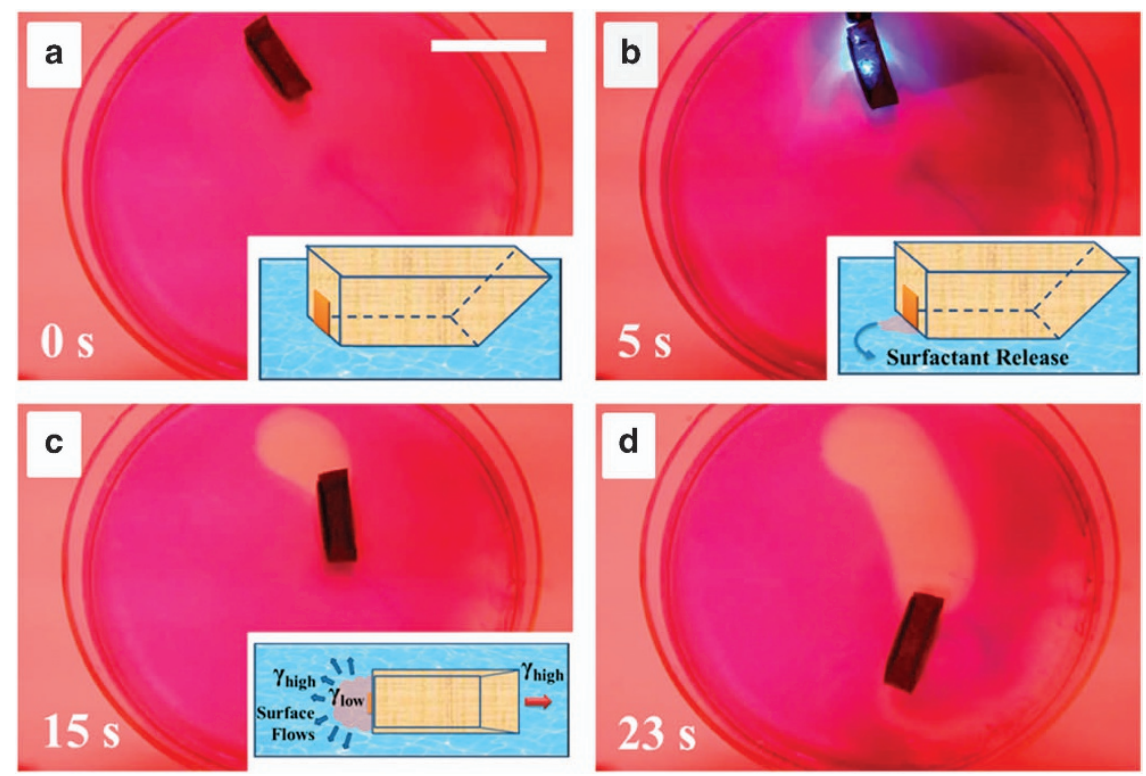

Figure 3 Photographs and corresponding schematic diagrams of the diffusion of the surfactant molecules released from the smart microactuator with thymol blue as an indicator. (a) The device remained motionless on the water surface; (b) surfactant was released after irradiation with ultraviolet (UV) light, initiating the movement of the smart device; (c and $\mathbf{d}$ ) the circle of surfactant displacing the indicator grew at the back of the boat owing to the diffusion of the surfactant after irradiation with UV light. Scale bar $=5 \mathrm{~cm}$.

Considering the balance between the velocity and controllability of the ON-OFF-ON motion, we optimized the volume ratio of the surfactant at $11 \%$, which led to a high velocity with good controllability. This volume ratio was used in all subsequent experiments, unless otherwise stated.

The etched depth of the photoresist layers determines the dissolution and diffusion of the surfactant on the water surface. Therefore, we used atomic force microscopy to investigate the photoreaction and developing kinetics of the photosensitive smart surface at a concentration of $11 \%$. Figure $2 \mathrm{c}$ plots the photoreaction of the photoresist irradiated with 365-nm UV light versus the exposure dose; the result is a typical reaction kinetics curve. The etched depth of the photoresist layers increased with the exposure dose and reached a constant when the exposure dose was $\sim 148 \mathrm{~mJ} \mathrm{~cm}^{-2}$ because the thickness of the photoresist coating was $\sim 1750 \mathrm{~nm}$ (Supplementary Figure S5) and was fully exposed at this irradiation dose. This finding suggests that to realize ON-OFF-ON motion, the initial exposure dose should be lower than $148 \mathrm{~mJ} \mathrm{~cm}^{-2}$ to avoid running out of surfactant.

The relationship between the etching depth of the photoresist layers and the developing time reflects the diffusion rate of the surfactant, which determines the motion velocity of the device. From Figure 2d, we can determine the relationship between the etched depth and developing time with the UV exposure dose fixed at $148 \mathrm{~mJ} \mathrm{~cm}{ }^{-2}$. Before the developing time reached $30 \mathrm{~s}$, the etched depth of the photoresist layers increased rapidly with increasing developing time; after $110 \mathrm{~s}$, the etched depth reached a constant because all of the exposed photoresist had been removed. This result indicated that the movement of the device was initiated when the photosensitive microactuator was exposed to $365-\mathrm{nm}$ UV light and stopped once the exposed mixture was washed off completely.

After we realized that the volume ratio of the surfactant and the exposure dose affected the locomotive behavior of the smart device, we wondered whether we could achieve ON-OFF-ON motion of the as-prepared smart device. Therefore, we performed an experiment in a trough $\left(34 \times 27 \mathrm{~cm}^{2}\right)$ with a smart device weighing $\sim 600 \mathrm{mg}$ and the irradiation intensity set at $17.5 \mathrm{~mW} \mathrm{~cm}^{-2}$ while changing the irradiation time to control its motion. As illustrated in Figure 4, there were two identical smart devices on the water surface. To demonstrate that the smart device could start only with UV irradiation instead of spontaneous motion, we exposed the upper device to UV light while keeping the lower device in the dark. As Figures $4 \mathrm{a}-\mathrm{c}$ demonstrate, when the upper device was irradiated by UV light, it ran at an average speed of $\sim 1.3 \pm 0.1 \mathrm{~cm} \mathrm{~s}^{-1}$ owing to the surface tension gradient caused by the released surfactant. When the exposure of the photoresist mixture was stopped, the device stopped because decomposition of the photoresist was paused without UV irradiation, surfactant release was stopped and the local surface tension gradient changed little. The motion was restarted by exposing the microactuator to UV light a second time (Figures $4 \mathrm{~d}$ and e), that is, an $\mathrm{ON}$ OFF-ON process was achieved owing to the surface tension gradient by release of surfactant after decomposing the photoresist. The moving velocity decreased to $0.9 \pm 0.1 \mathrm{~cm} \mathrm{~s}^{-1}$ because the surface tension gradient in the second UV exposure was lower than that in the first irradiation. When we irradiated the microactuator for a third time, the device remained motionless because all of the photoresist had decomposed and the surfactant had been washed off, leaving no fuel for propulsion. During the ON-OFF-ON of the upper device, the lower device remained still, which supported our hypothesis that only a UV stimulus could start the motion. To further confirm this conclusion, we started the lower device by exposing it to UV irradiation (Figures $4 \mathrm{f}$ and $\mathrm{g}$; to see the entire process, see Supplementary Movie S3).

To show the ON-OFF-ON motion more clearly, we performed a similar experiment in a small rectangular glass trough $\left(24 \times 2.7 \mathrm{~cm}^{2}\right)$ and recorded the locomotive behavior from the side (Figure 5 and Supplementary Movie S4). Before UV irradiation, the device was motionless on the solution surface; after the photosensitive microactuator was exposed to UV light $\left(17.5 \mathrm{~mW} \mathrm{~cm}^{-2}\right)$ for $3 \mathrm{~s}$, the device 

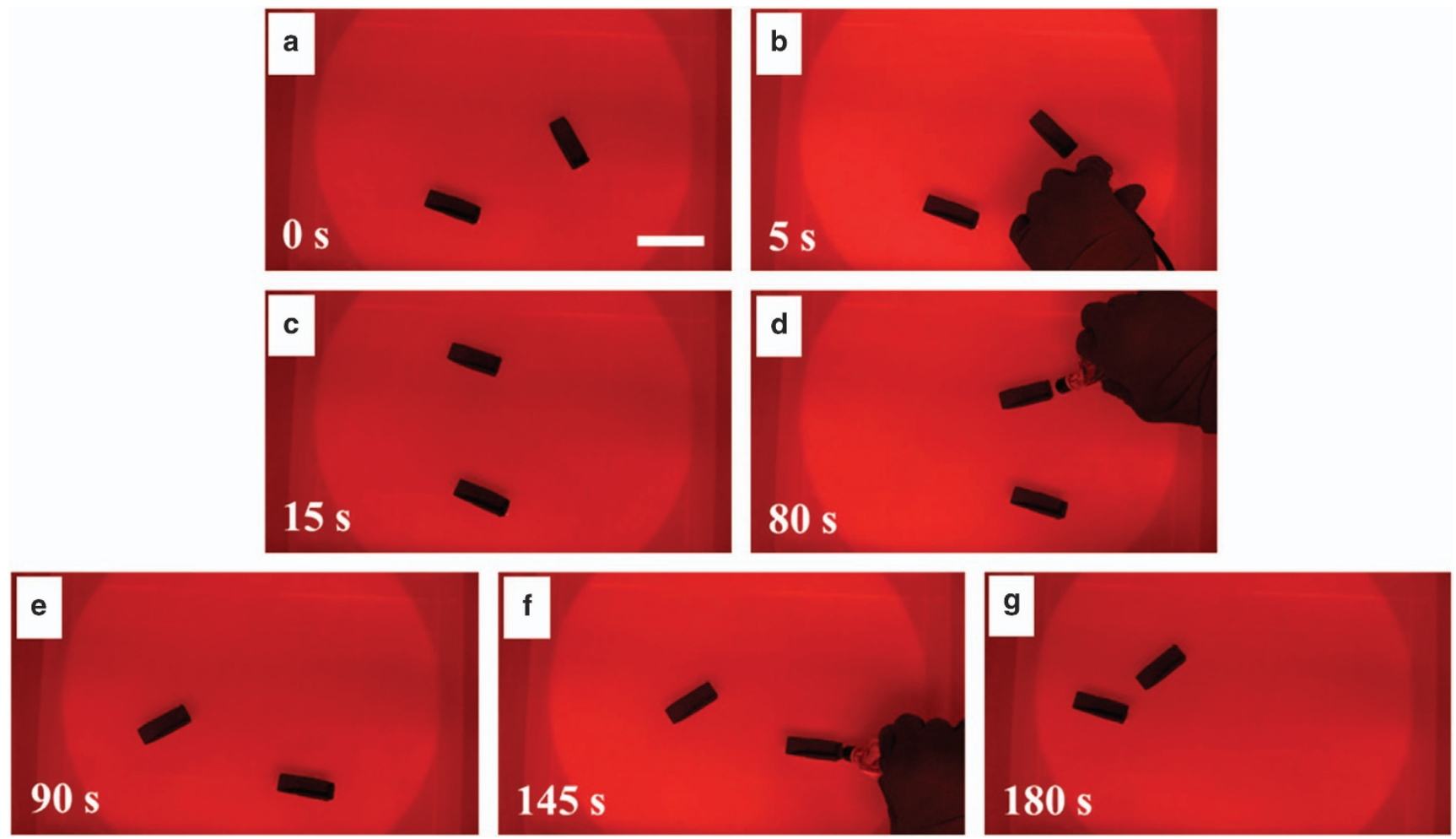

Figure 4 Photographs of the moving process of two identical smart devices. (a-e) The upper device moved after ultraviolet (UV) irradiation, whereas the bottom device remained still in the dark. ( $\mathbf{f}$ and $\mathbf{g}$ ) The bottom device moved after being exposed to UV irradiation. Scale bar $=5 \mathrm{~cm}$.
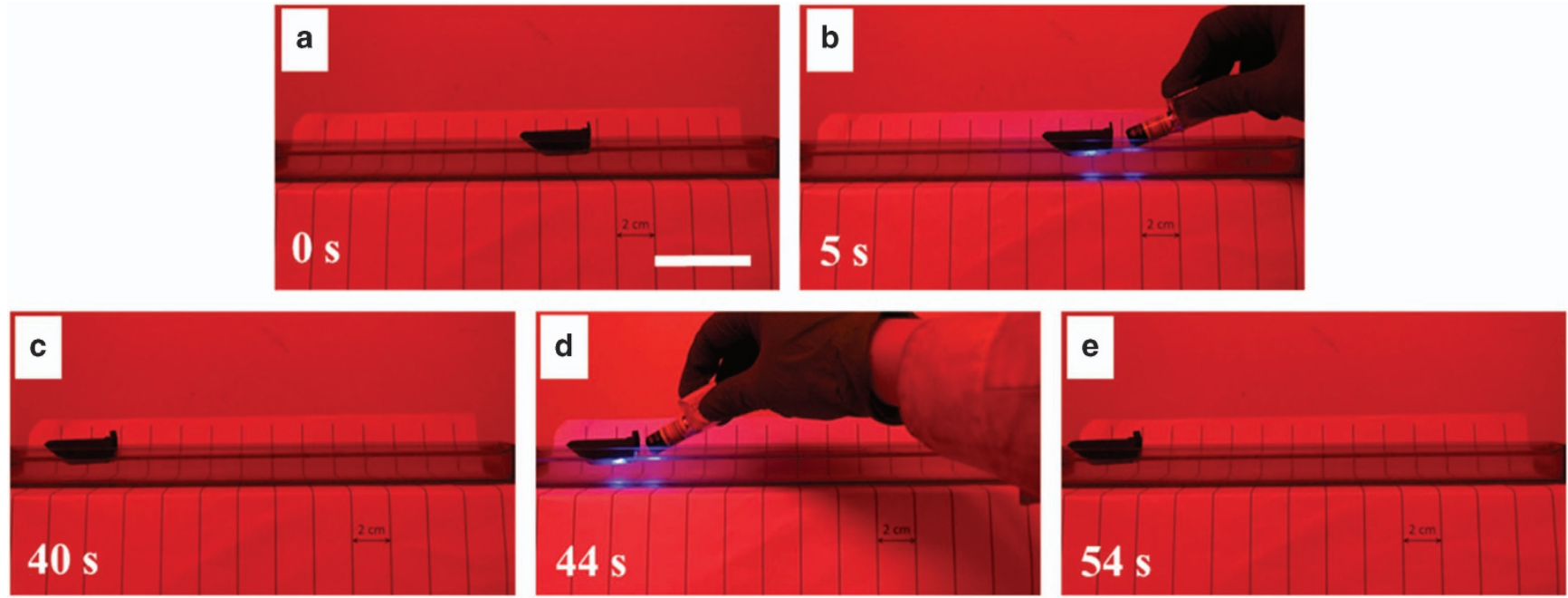

Figure 5 Photographs of the locomotion of the smart device from a side view in a rectangular glass trough. Scale bar $=5 \mathrm{~cm}$.

started to run at an average speed of $1 \mathrm{~cm} \mathrm{~s}^{-1}$ for $14 \mathrm{~s}$ and stopped once the exposed portion of the mixture was washed off. The speed was slower in this trough than in the larger trough because the smaller surface area of the water surface decreased the space over which the surfactant diffused, leading to the aggregation of surfactant molecules and reducing the surface tension gradient. After exposure to UV light for a second time, the device ran at a slower speed for another $5 \mathrm{~s}$ until it reached the end of the glass trough. This result occurred because the gradient of the surface tension after the second UV exposure decreased markedly compared with that after the first exposure, resulting in a reduced Marangoni effect.

As we had demonstrated that the device could move in a straight line with few deviations (the deviation angle was $< \pm 15^{\circ}$, and the incidence rate was below 30\%; for details, see Section S6 in Supplementary Information), we wondered whether we could control 

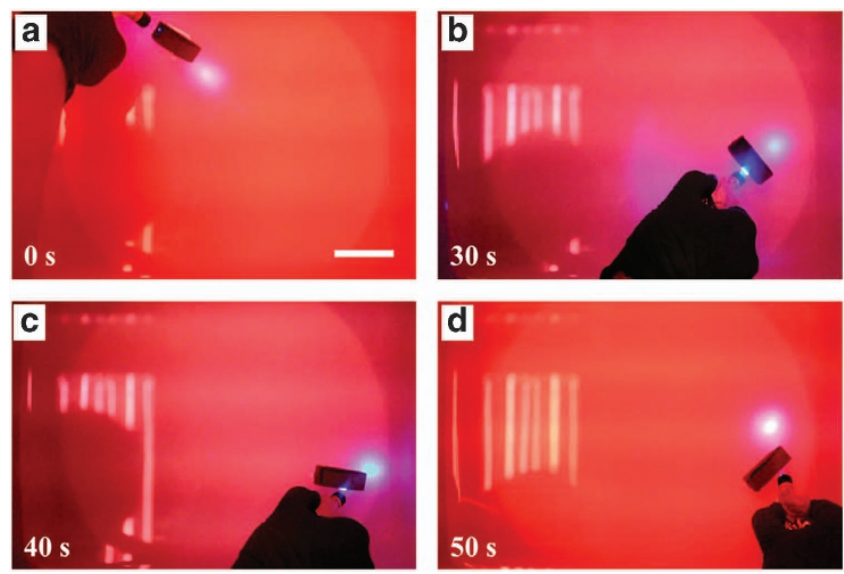

Figure 6 Photographs of the locomotion with direction selectivity of the smart device. Scale bar $=5 \mathrm{~cm}$.

the moving direction of the device. Therefore, we fabricated a new smart device attached with two photo-sensitive microactuators: one at the end of the device and the other on the right side of the superhydrophobic boat. As shown in Figure 6, initially, the device remained essentially motionless in the dark; when the photoresponsive microactuator at the end of the smart device was exposed to UV irradiation, the device began to run in a straight line; during its locomotion, the UV irradiation was applied to the microactuator on the right side of the device and the smart device turned left by an angle of $\sim 90^{\circ}$ (for the details, see Supplementary Movie S5). This result demonstrated that by designing the smart device with microactuators on different sides and selectively exposing these microactuators, we could control straight-line movement the moving direction of the smart device.

\section{CONCLUSIONS}

In conclusion, we achieved ON-OFF-ON motion of a smart device, which consisted of a superhydrophobic boat with drag-reducing properties and a UV-responsive microactuator. The as-prepared smart device started moving under UV irradiation, stopped after fully releasing the surfactant within the exposed mixture loaded on the microactuator and restarted locomotion when UV irradiation was reapplied on the microactuator. This ON-OFF-ON motion could be achieved for several cycles until all of the surfactant and photoresist in the mixture ran out. Moreover, by designing the smart device with micro-actuators at different locations and selectively exposing them to UV irradiation, we could not only achieve straight-line movement but could also adjust the moving direction. This work is the first example using the chemical Marangoni effect to produce photoresponsive motion on a water surface.

\section{CONFLICT OF INTEREST}

The authors declare no conflict of interest.

\section{ACKNOWLEDGEMENTS}

This work was supported by the NSFC (21374006), the Program of the CoConstruction with Beijing Municipal Commission of Education of China, the Fok Ying Tung Education Foundation (131013), the Open Project of State Key Laboratory of Supramolecular Structure and Materials (SKLSSM201401) and the Beijing Young Talents Plan (YETP0488).
$1 \mathrm{Hu}$, D. L., Chan, B. J. \& Bush, W. M. The hydrodynamics of water strider locomotion. Nature 424, 663-666 (2003).

2 Shi, F., Niu, J., Liu, J. L., Liu, F., Wang, Z. Q., Feng, X. Q. \& Zhang, X. Towards understanding why a superhydrophobic coating is needed by water striders. Adv. Mater. 19, 2257-2261 (2007).

3 Ozcan, O., Wang, H., Taylor, J. D. \& Sitti, M. Surface tension driven water strider robot using circular footpads. Proceedings of IEEE International Conference on Robotics and Automation 3799-3804 (Anchorage, Alaska, USA, 2010).

4 Ismagilov, R. F., Schwartz, A., Bowden, N. \& Whitesides, G. M. Autonomous movement and self-assembly. Angew. Chem. Int. Ed. 41, 652-654 (2002).

5 Shi, F., Liu, S. H., Gao, H. T., Ding, N., Dong, L. J., Tremel, W. \& Knoll, W. Magneticfield-induced locomotion of glass fibers on water surfaces: towards the understanding of how much force one magnetic nanoparticle can deliver. Adv. Mater. 21, 1927-1930 (2009).

6 Dong, H. Y., Cheng, M. J., Zhang, Y. J., Wei, H. \& Shi, F. Extraordinary drag-reducing effect of a superhydrophobic coating on a macroscopic model ship at high speed. J. Mater. Chem. A 1, 5886-5891 (2013).

7 Chang, S. T., Paunov, V. N., Petsev, D. N. \& Velev, O. D. Remotely powered self-propelling particles and micropumps based on miniature diodes. Nat. Mater. 6, 235-240 (2007)

8 Cheng, M. J., Gao, H. T., Zhang, Y. J., Tremel, W., Chen, J. F., Shi, F. \& Knoll, W. Combining magnetic field induced locomotion and supramolecular interaction to micromanipulate glass fibers: toward assembly of complex structures at mesoscale. Langmuir 27, 6559-6564 (2011)

9 Wilson, D. A., Nolte, R. J. M. \& Hest van, J. C. M. Autonomous movement of platinumloaded stomatocytes. Nat. Chem. 4, 268-274 (2012).

10 Solovev, A. A., Sanchez, S., Pumera, M., Mei, Y. F. \& Schmidt, O. G. Magnetic control of tubular catalytic microbots for the transport, assembly, and delivery of micro-objects. Adv. Funct. Mater. 20, 2430-2435 (2010).

11 Solovev, A. A., Xi, W., Gracias, D. H., Harazim, S. M., Deneke, C., Sanchez, S. \& Schmidt, O. G. Self-propelled nanotools. ACS Nano 6, 1751-1756 (2012).

12 Gao, W., Sattayasamitsathit, S., Orozco, J. \& Wang, J. Highly efficient catalytic microengines: template electrosynthesis of polyaniline/platinum microtubes. J. Am. Chem. Soc. 133, 11862-11864 (2011).

13 Bidoz, S. F., Arsenault, A. C., Manners, I. \& Ozin, G. A. Synthetic self-propelled nanorotors. Chem. Commun. 441-443 (2005).

14 Kline, T. R., Paxton, W. F., Mallouk, T. E. \& Sen, A. Catalytic nanomotors: remotecontrolled autonomous movement of striped metallic nanorods. Angew. Chem. Int. Ed. 44, 744-746 (2005).

15 Paxton, W. F., Sen, A. \& Mallouk, T. E. Motility of catalytic nanoparticles through selfgenerated forces. Chem. Eur. J. 11, 6462-6470 (2005).

16 Howse, J. R., Jones, R. A. L., Ryan, A. J., Gough, T., Vafabakhsh, R. \& Golestanian, R. Self-motile colloidal particles: from directed propulsion to random walk. Phys. Rev. Lett. 99, 048102 (2007)

17 Wu, Z. G., Wu, Y. J., He, W. P., Lin, X. K., Sun, J. M. \& He, Q. Self-propelled polymerbased multilayer nanorockets for transportation and drug release. Angew. Chem. Int. Ed. 52, 7000-7003 (2013).

18 Gao, Y. F., Cheng, M. J., Wang, B. L., Feng, Z. G. \& Shi, F. Diving-surfacing cycle within a stimulus-responsive smart device towards developing functionally cooperating systems. Adv. Mater. 22, 5125-5128 (2010).

19 Ju, G. N., Cheng, M. J., Xiao, M., Xu, J. M., Pan, K., Wang, X. Y., Zhang, J. \& Shi, F. Smart transportation between three phases through a stimulus-responsive functionally cooperating device. Adv. Mater. 25, 2915-2919 (2013).

20 Gao, W., Uygun, A. \& Wang, J. Hydrogen-bubble-propelled zinc-based microrockets in strongly acidic media. J. Am. Chem. Soc. 134, 897-900 (2012).

21 Mou, F. Z., Chen, C. R., Ma, H. R., Yin, Y. X., Wu, Q. Z. \& Guan, J. G. Self-propelled micromotors driven by the magnesium-water reaction and their hemolytic properties. Angew. Chem. 125, 7349-7353 (2013)

22 Lagzi, I., Soh, S., Wesson, P. J., Browne, K. P. \& Grzybowski, B. A. Maze solving by chemotactic droplets. J. Am. Chem. Soc. 132, 1198-1199 (2010).

23 Zhang, H., Duan, W. T., Liu, L. \& Sen, A. Depolymerization-powered autonomous motors using biocompatible fuel. J. Am. Chem. Soc. 135, 15734-15737 (2013).

24 Xiao, M., Cheng, M. J., Zhang, Y. J. \& Shi, F. Combining the Marangoni effect and the $\mathrm{pH}$-responsive superhydrophobicity-superhydrophilicity transition to biomimic the locomotion process of the beetles of genus Stenus. Small 9, 2509-2514 (2013).

25 Schmidt, J. J. \& Montemagno, C. D. Bionanomechanical systems. Annu. Rev. Mater. Res. 34, 315-337 (2004).

26 Schildknecht, H. Chemical ecology - a chapter of modern natural products chemistry. Angew. Chem. Int. Ed. Engl. 15, 214-222 (1976).

27 Bush, J. W. M., Hu, D. L. \& Prakash, M. The integument of water-walking arthropods: form and function. Adv. Insect Physiol. 34, 117-192 (2007).

28 Scriven, L. E. \& Sternling, C. V. The Marangoni effects. Nature 187, 186-188 (1960).

29 Takabatake, F., Magome, N., Ichikawa, M. \& Yoshikawa, K. Spontaneous modeselection in the self-propelled motion of a solid/liquid composite driven by interfacial instability. J. Chem. Phys. 134, 114704 (2011).

30 Sharma, R., Chang, S. T. \& Velev, O. D. Gel-based self-propelling particles get programmed to dance. Langmuir 28, 10128-10135 (2012).

31 Lauga, E. \& Davis, A. M. J. Viscous Marangoni propulsion. J. Fluid Mech. 705, 120-133 (2012)

32 Magome, N. \& Yoshikawa, K. Nonlinear oscillation and ameba-like motion in an oil/ water system. J. Phys. Chem. 100, 19102-19105 (1996). 
33 Zhao, G. J., Seah, T. H. \& Pumera, M. External-energy-independent polymer capsule motors and their cooperative behaviors. Chem. Eur. J. 17, 12020-12026 (2011).

34 Nakata, S., Kirisaka, J., Arima, Y. \& Ishii, T. Self-Motion of a camphanic acid disk on water with different types of surfactants. J. Phys. Chem. B 110, 21131-21134 (2006).

35 Xiao, M., Guo, X. P., Cheng, M. J., Ju, G. N., Zhang, Y. J. \& Shi, F. pH-responsive on-off motion of a superhydrophobic boat: towards the design of a minirobot. Small 10 859-865 (2014).

36 Okawa, D., Pastine, S. J., Zettl, A. \& Fréchet, J. M. J. Surface tension mediated conversion of light to work. J. Am. Chem. Soc. 131, 5396-5398 (2009).

37 Diguet, A., Guillermic, R. M., Magome, N., Jalmes, A. S., Chen, Y., Yoshikawa, K. \& Baigl, D. Photomanipulation of a droplet by the chromocapillary effect. Angew. Chem. Int. Ed. 48, 9281-9284 (2009).

38 Diguet, A., Li, H., Queyriaux, N., Chen, Y. \& Baigl, D. Photoreversible fragmentation of a liquid interface for micro-droplet generation by light actuation. Lab Chip 11, 2666-2669 (2011)

39 Cassie, A. B. D. \& Baxter, S. Wettability of porous surfaces. Trans. Faraday Soc. 40, 546-551 (1944).
40 Zhang, X., Shi, F., Yu, X., Liu, H., Fu, Y., Wang, Z. Q., Jiang, L. \& Li, X. Y. Polyelectrolyte multilayer as matrix for electrochemical deposition of gold clusters: toward super-hydrophobic surface. J. Am. Chem. Soc. 126, 3064-3065 (2004).

41 Ward, A. F. H. Thermodynamics of monolayers on solutions. Trans. Faraday Soc. 399-407 (1946).

(c) (i) (2)(2) This work is licensed under a Creative Commons Attribution-NonCommercial-ShareAlike 3.0 Unported License. The images or other third party material in this article are included in the article's Creative Commons license, unless indicated otherwise in the credit line; if the material is not included under the Creative Commons license, users will need to obtain permission from the license holder to reproduce the material. To view a copy of this license, visit http://creativecommons.org/licenses/by-nc-sa/3.0/

Supplementary Information accompanies the paper on the NPG Asia Materials website (http://www.nature.com/am) 